\title{
WEAK APPROXIMATION OVER FUNCTION FIELDS
}

by

\author{
Brendan Hassett and Yuri Tschinkel
}

ABstract. - We prove that rationally connected varieties over the function field of a complex curve satisfy weak approximation for places of good reduction.

\section{Contents}

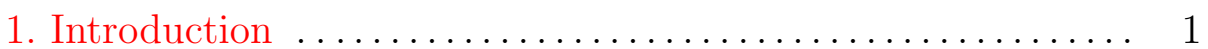

2. Basic properties of weak approximation $\ldots \ldots \ldots \ldots \ldots \ldots$

3. Rationally connected varieties $\ldots \ldots \ldots \ldots \ldots \ldots \ldots \ldots$

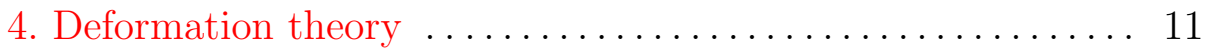

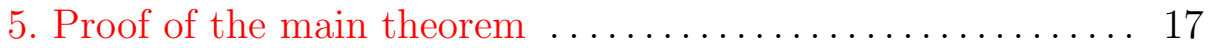

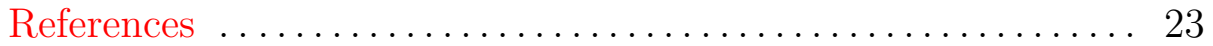

\section{Introduction}

Let $F$ be a number field and $X$ an algebraic variety over $F$. Does there exist an $F$-rational point on $X$ ? If so, are they ubiquitous on $X$ ? For many classes of varieties, such problems are analyzed using local-to-global principles. The variety $X$ is said to satisfy the Hasse principle if existence of a rational point over each completion of $F$ implies the existence of an $F$-rational point. The variety $X$ is said to satisfy the principle of weak approximation if for every finite set of places of $F$ and points of $X$ over these places, there exists an $F$-rational point of $X$ approximating these points arbitrarily closely. 
Now let $k$ be an algebraically closed field of characteristic zero, $F$ the function field of a curve over $k$, and $B$ a smooth projective model for this curve. The following result by Graber, Harris and Starr is the impetus for our paper:

THEOREM 1 ([9],Theorem 1.2). - Every geometrically rationally connected variety $X$ over $F$ has an $F$-rational point.

An algebraic variety is rationally connected if any two points can be joined by a rational curve (see Section 3.1 for more details). Rational and unirational varieties are rationally connected. We refer the reader to [10] for related results in positive characteristic (see also Remark 28).

From an arithmetic viewpoint, proving such a theorem entails surmounting two obstacles: First there must be no obstructions to the existence of a local point, i.e., $X\left(F_{\nu}\right) \neq \emptyset$ for all completions $F_{\nu}$ of $F$. If $X$ is proper with a model $\pi: \mathcal{X} \rightarrow B$ proper and flat over $B$, this corresponds to producing formal sections of $\pi$ at each point $b \in B$. Second, the Hasse principle must hold for $X$ over $F$, i.e., $X(F) \neq \emptyset$. Note that over a number field, the Hasse principle may fail even for cubic surfaces. When $X$ is proper with model as above, an $F$-rational point of $X$ corresponds to a section of $\pi$.

Theorem 1 naturally leads one to pose the following

CONJECTURE 2. - A smooth geometrically rationally connected variety $X$ over $F$ satisfies weak approximation.

This has a nice geometric interpretation when $X$ admits a proper model $\pi: \mathcal{X} \rightarrow B$ : There exist sections with prescribed jets over a finite number of points of $B$ (see Section 2 for more discussion). Our main result is weak approximation at places of good reduction, i.e., away from singular fibers:

THEOREM 3. - A smooth proper geometrically rationally connected variety $X$ over $F$ satisfies weak approximation for places of good reduction.

Kollár, Miyaoka, and Mori established the zeroth-order case, assuming the existence of at least one section: 
Theorem 4 ([11] IV.6.10, [12] 2.13). — Let $X$ be a smooth projective geometrically rationally connected variety over $F$ and $\pi: \mathcal{X} \rightarrow B$ a projective model. Given a finite collection of points $\left\{b_{i}\right\}_{i \in I}$ such that each fiber $\mathcal{X}_{b_{i}}$ is smooth and points $x_{i} \in \mathcal{X}_{b_{i}}$, there exists a section $s: B \rightarrow \mathcal{X}$ such that $s\left(b_{i}\right)=x_{i}$, for each $i \in I$.

It is natural to try to relax the hypothesis that the fibers $\mathcal{X}_{b_{i}}$ be smooth. Suppose that the total space $\mathcal{X}$ of our model is regular. Then for each section $s, s(b) \in \mathcal{X}_{b}$ is necessarily a smooth point; otherwise, the intersection multiplicity of $s(B)$ with $\mathcal{X}_{b}$ would be $>1$. In light of this, an optimistic generalization of Theorem 4 would be:

Conjecture 5. - Let $X$ be a smooth proper geometrically rationally connected variety over $F$ and $\pi: \mathcal{X} \rightarrow B$ a regular proper model. Given a finite collection of points $\left\{b_{i}\right\}_{i \in I} \subset B$ and smooth points $r_{i} \in \mathcal{X}_{b_{i}}$, there exists a section $s: B \rightarrow \mathcal{X}$ such that $s\left(b_{i}\right)=r_{i}$ for each $i \in I$.

This is obviously a consequence of Conjecture 2, and is in fact equivalent to it (see Remark 12).

For varieties over the function field $F$ of a curve, weak approximation is satisfied in the following cases [7]:

- varieties stably rational over $F$;

- connected linear algebraic groups over $F$ and homogeneous spaces for these groups;

- homogeneous space fibrations over varieties that satisfy weak approximation, e.g., conic bundles over varieties rational over $F$;

- Del Pezzo surfaces of degree at least four.

Weak approximation is not known for general cubic surfaces. Madore proved weak approximation for cubic surfaces away from places of bad reduction [13].

Acknowledgments: The first author was partially supported by the Sloan Foundation and NSF Grants 0134259 and 0196187. The second author was partially supported by NSF Grant 0100277. Part of this work was done while both authors were visiting the American Institute of Mathematics in Palo Alto. We thank J. L. Colliot-Thélène, J. de Jong, O. Gabber, T. Graber, J. Harris, J. Kollár and J. Starr for conversations about this topic. 


\section{Basic properties of weak approximation}

2.1. Definition. - Let $F$ be a number field or a function field of a smooth projective curve $B$ over an algebraically closed ground field $k$. For each place $\nu$ of $F$, let $F_{\nu}$ denote the $\nu$-adic completion of $F$. Let $X$ be an algebraic variety of dimension $d$ over $F$; in this paper, all varieties are assumed to be geometrically integral. Let $X(F)$ denote the set of $F$-rational points of $X$. One says that rational points on $X$ satisfy weak approximation if, for any finite set of places $\left\{\nu_{i}\right\}_{i \in I}$ of $F$ and $\nu_{i}$-adic open subsets $U_{i} \subset X\left(F_{\nu_{i}}\right)$, there is a rational point $x \in X(F)$ such that its image in each $X\left(F_{\nu_{i}}\right)$ is contained in $U_{i}$. In particular, for any collection of $x_{i} \in X\left(F_{\nu_{i}}\right), i \in I$, there exists an $x \in X(F)$ arbitrarily close to each $x_{i}$.

It is well known that weak approximation is a birational property: If $X_{1}$ and $X_{2}$ are smooth varieties birational over $F$ then $X_{1}$ satisfies weak approximation if and only if $X_{2}$ satisfies weak approximation.

Over a field of characteristic zero, weak approximation for smooth varieties reduces to the case of weak approximation for smooth projective varieties: Chow's lemma and resolution of singularities imply the existence of a smooth projective variety birational to any variety.

For the rest of this paper we restrict our attention to the function field case. Places $\nu$ of $F$ correspond to points $b$ on $B$. Let $X$ be a smooth proper variety over $F$.

We discuss geometric formulations of weak approximation when $X$ admits a proper model

$$
\pi: \mathcal{X} \rightarrow B
$$

i.e., a flat proper morphism with generic fiber $X$. If $X$ is projective then the properness of the Hilbert scheme gives such a model over $B$; if $X$ is proper then such model exists by a result of Nagata [15]. For each $b \in B$, let

$$
\mathcal{X}_{b}=\mathcal{X} \times_{B} \operatorname{Spec}\left(\mathcal{O}_{B, b} / \mathfrak{m}_{B, b}\right)
$$

denote the fiber over $b$. Sections of $\pi$ yield $F$-valued points of $X$ and conversely, each $F$-valued point of $X$ extends to a section of $\pi$. Let $\widehat{B}_{b}$ denote the completion of $B$ at $b$ and $\left(\widehat{\mathcal{O}}_{B, b}, \widehat{\mathfrak{m}}_{B, b}\right)$ the associated complete local ring, which has fraction field $F_{\nu}$. Restricting to this formal neighborhood of $b$ gives 


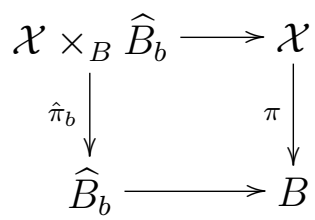

Sections of $\hat{\pi}_{b}$ restrict to $F_{\nu}$-valued points of $X$ and conversely, each $F_{\nu^{-}}$ valued point of $X$ extends to a section of $\hat{\pi}_{b}$. Basic $\nu$-adic open subsets of $X\left(F_{\nu}\right)$ consist of those sections of $\hat{\pi}_{b}$ which agree with a given section over $\operatorname{Spec}\left(\widehat{\mathcal{O}}_{B, b} / \widehat{\mathfrak{m}}_{B, b}^{N+1}\right) \subset \widehat{B}_{b}$, for some $N \in \mathbb{N}$. Weak approximation means that for any finite set of points $\left\{b_{i}\right\}_{i \in I}$ in $B$, sections $\hat{s}_{i}$ of $\hat{\pi}_{b_{i}}$, and $N \in \mathbb{N}$, there exists a section $s$ of $\pi$ agreeing with $\hat{s}_{i}$ modulo $\widehat{\mathfrak{m}}_{B, b_{i}}^{N+1}$ for each $i$.

2.2. Fibers of good reduction. - A place $\nu$ is of good reduction for $X$ if there exists an algebraic space

$$
\widehat{\mathcal{X}}_{b} \rightarrow \widehat{B}_{b}
$$

proper and smooth over $\widehat{B}_{b}$, with generic fiber isomorphic to $X$ over $F_{\nu}$. Let $S$ denote the finite set of places of bad reduction.

Definition 6. - A proper variety $X$ satisfies weak approximation for places of good reduction if, for any finite set of places of good reduction $\left\{\nu_{i}\right\}_{i \in I}$ and $\nu_{i}$-adic open subsets $U_{i} \subset X\left(F_{\nu_{i}}\right)$, there is a rational point $x \in X(F)$ such that its image in each $X\left(F_{\nu_{i}}\right)$ is contained in $U_{i}$.

Proposition 7. - Suppose $X$ is a smooth proper variety over F. There exists an algebraic space

$$
\pi: \mathcal{X} \rightarrow B
$$

proper and flat over $B$, smooth over the places of good reduction, and with generic fiber $X$. Such a space is called a good model of $X$ over $B$.

Proof. - Choose a proper model $\pi^{\prime}: \mathcal{X}^{\prime} \rightarrow B$ for $X$ over $B$ [15]. If $\pi^{\prime}$ is smooth over $B \backslash S$ there is nothing to prove. Otherwise, let $\left\{b_{j}\right\} \subset B \backslash S$ denote the points over which $\mathcal{X}_{b_{j}}^{\prime}$ is singular; let $\widehat{\mathcal{X}}_{b_{j}}^{\prime}$ denote the completion of $\mathcal{X}^{\prime}$ along the central fiber $\mathcal{X}_{b_{j}}^{\prime}$. By assumption, there exists a proper smooth space

$$
\widehat{\mathcal{X}}_{b_{j}} \rightarrow \widehat{B}_{b_{j}}
$$


which is isomorphic to our original model over the generic point. Resolving the indeterminacy of the rational map

$$
\widehat{\mathcal{X}}_{b_{j}} \rightarrow-\widehat{\mathcal{X}}_{b_{j}}^{\prime}
$$

we find that these are related by a sequence of modifications in the central fiber. This gives a sequence of formal modifications to $\mathcal{X}^{\prime}$ along the singular fibers, in the sense of Artin [3]. Theorems 3.1 and 3.2 of [3] give a unique proper algebraic space $\pi: \mathcal{X} \rightarrow B$ realizing these formal modifications to $\mathcal{X}^{\prime}$.

EXAMPLE 8. - There are simple examples justifying the introduction of algebraic spaces. Let $\pi: \mathcal{X}^{\prime} \rightarrow B$ be a flat projective morphism such that each fiber is a cubic surface with rational double points and the generic fiber $X$ is smooth. Suppose that near each point $b \in B$ the local monodromy representation

$$
\operatorname{Gal}\left(\bar{F}_{\nu} / F_{\nu}\right) \rightarrow \operatorname{Aut}(\operatorname{Pic}(\bar{X}))
$$

is trivial. By a theorem of Brieskorn [4] [5], there exists a simultaneous resolution

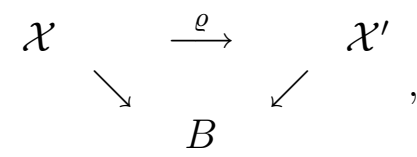

where $\mathcal{X}$ is a smooth proper algebraic space over $B$ and $\varrho_{b}: \mathcal{X}_{b} \rightarrow \mathcal{X}_{b}^{\prime}$ is the minimal resolution of $\mathcal{X}_{b}^{\prime}$ for each $b \in B$. However, $\mathcal{X}$ is constructed by making modifications of $\mathcal{X}^{\prime}$ in formal neighborhoods of the singular fibers, and hence is not necessarily a scheme. Note that blowing up the singularities of $\mathcal{X}^{\prime}$ will usually introduce exceptional divisors in the fibers.

\subsection{Jets and iterated blowups. -}

Definition 9. - Let $\pi: \mathcal{X} \rightarrow B$ be a proper model. An admissible $N$-jet of $\pi$ at $b$ is a section of

$$
\mathcal{X} \times_{B} \operatorname{Spec}\left(\mathcal{O}_{B, b} / \mathfrak{m}_{B, b}^{N+1}\right) \rightarrow \operatorname{Spec}\left(\mathcal{O}_{B, b} / \mathfrak{m}_{B, b}^{N+1}\right)
$$

whose image is a smooth point of $\mathcal{X}_{b}$.

Hensel's lemma guarantees that every admissible $N$-jet is the restriction of a section of $\hat{\pi}_{b}$. When $\pi$ is a good model and $b$ is of good reduction then every $N$-jet at $b$ is admissible. Thus weak approximation at places 
of good reduction is equivalent to showing there exist sections with prescribed jets over a finite set of places in $B \backslash S$.

REMARK 10. - Here is a tempting (but false) argument for Theorem 3. Let $\mathcal{Y} \rightarrow B$ be the space of all $N$-jets for $\mathcal{X}$; over the places of good reduction, $\mathcal{Y}$ is an affine-space bundle over $\mathcal{X}$. Let $\psi: \overline{\mathcal{Y}} \rightarrow B$ be some compactification of $\mathcal{Y}$ with smooth fibers at places of good reduction. An $N$-jet at $b_{i}$ corresponds to a point $j_{i} \in \overline{\mathcal{Y}}_{b_{i}}$. Theorem 4 gives a section $t$ of $\psi$ through the $j_{i}$, which projects back down to a section $s$ of $\pi$.

The problem is that $t$ is not integrable, i.e., it need not coincide with the natural lift of $s$. Thus $s$ may not have the desired jet data.

Let $\pi: \mathcal{X} \rightarrow B$ be proper model of $X,\left\{b_{i}\right\}$ a finite collection of distinct points of $B$, and $J=\left\{j_{i}\right\}_{i \in I}$ admissible $N$-jets at these points. The iterated blowup associated with $J$

$$
\beta(J): \mathcal{X}(J) \rightarrow \mathcal{X}
$$

is obtained by performing the following sequence of blowups: For each $i \in I$ choose a section $\hat{s}_{i}$ of $\hat{\pi}_{b_{i}}$ with jet $j_{i}$. Now blow up $\mathcal{X}$ successively $N$ times, where at each stage the center is the point at which the proper transform of $\hat{s}_{i}$ meets the fiber over $b_{i}$. Observe that a blowup of $\mathcal{X}$ centered in the fibers of $\pi$ is uniquely determined by the corresponding blowup of the completions along those fibers. Note that at each stage we blow up a smooth point of the fiber of the corresponding model and that the result does not depend on the order of the $b_{i}$ or on the choice of $\hat{s}_{i}$.

The fiber $\mathcal{X}(J)_{b_{i}}$ decomposes into irreducible components

$$
\mathcal{X}(J)_{b_{i}}=E_{i, 0} \cup \ldots \cup E_{i, N}
$$

where

- $E_{i, 0}$ is the proper transform of $\mathcal{X}_{b_{i}}$, isomorphic to the blowup of $\mathcal{X}_{b_{i}}$ at $r_{i, 0}:=\hat{s}_{i}\left(b_{i}\right)$;

- $E_{i, n}, n=1, \ldots, N-1$, is the blowup of $\mathbb{P}^{d}$ at $r_{i, n}$, the point where the proper transform of $\hat{s}_{i}$ meets the fiber over $b_{i}$ of the $n$th blowup;

$-E_{i, N} \simeq \mathbb{P}^{d}$.

The intersection $E_{i, n} \cap E_{i, n+1}$ is the exceptional divisor $\mathbb{P}^{d-1} \subset E_{i, n}$ and a proper transform of a hyperplane in $E_{i, n+1}$, for $n=0, \ldots, N-1$. Let $r_{i} \in E_{i, N} \backslash E_{i, N-1}$ denote the intersection of $\hat{s}_{i}$ with $E_{i, N}$. 


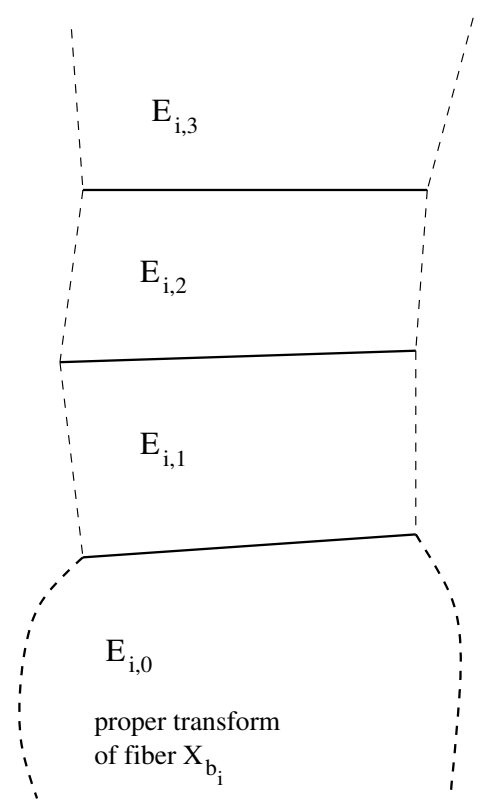

Figure 1. One fiber of the iterated blowup

For each section $s^{\prime}: \mathcal{X}(J) \rightarrow B$ the composition $\beta(J) \circ s^{\prime}$ is a section of $\pi: \mathcal{X} \rightarrow B$. Conversely, given a section $s$ of $\pi: \mathcal{X} \rightarrow B$, its proper transform $s^{\prime}$ is the unique section of $\mathcal{X}(J) \rightarrow B$ lifting $s$. Sections $s^{\prime}$ of $\mathcal{X}(J) \rightarrow B$ with $s^{\prime}\left(b_{i}\right)=r_{i}$ yield sections of $\mathcal{X} \rightarrow B$ with $N$-jet $j_{i}$ at $b_{i}$. We therefore have the following criterion for weak approximation in fibers of good reduction:

Proposition 11. - Let $\pi: \mathcal{X} \rightarrow B$ be a good model of $X$. Consider all data consisting of collections of points of good reduction $\left(b_{i}\right)_{i \in I}$ and $N$ jets $J=\left(j_{i}\right)$ over these points, and points on the corresponding iterated blowup $\mathcal{X}(J),\left(r_{i} \in E_{i, N} \backslash E_{i, N-1}\right)_{i \in I}$. If for every datum there exists a section $s^{\prime}$ of $\mathcal{X}(J) \rightarrow B$ with $s^{\prime}\left(b_{i}\right)=r_{i}$ for each $i \in I$, then $X$ satisfies weak approximation at places of good reductions.

REMARK 12. - Observe that Conjecture 2 follows from Conjecture 5, in characteristic zero. Weak approximation is a birational property of smooth varieties, so it suffices to prove Conjecture 2 for all projective smooth varieties. Fix a proper model $\pi: \mathcal{X} \rightarrow B$ with $\mathcal{X}$ regular. The 
argument for Proposition 11 reduces weak approximation for $\pi$ to proving the existence of sections passing through suitable points on iterated blowups of $\mathcal{X}$. Conjecture 5 asserts the existence of such sections.

\section{Rationally connected varieties}

Here the ground field $k$ is algebraically closed of characteristic zero.

3.1. Terminology and fundamental results. - Rational connectedness was introduced in the classification of Fano varieties [6] [12]. However, rationally connected varieties are now of independent interest:

Definition 13 ([11] IV.3.2). - A variety $Y$ is rationally chain connected (resp. rationally connected) if there is a family of proper and connected curves $g: U \rightarrow Z$ whose geometric fibers have only rational components (resp. are irreducible rational curves) and a cycle morphism $g: U \rightarrow Y$ such that

$$
u^{(2)}: U \times{ }_{Z} U \rightarrow Y \times Y .
$$

is dominant.

Our definition of 'rationally chain connected' makes sense for reducible schemes $Y$.

EXAMPLE 14. - The class of rationally connected varieties includes unirational varieties and smooth Fano varieties, [11] IV.3.2.6, V.2.13. In particular, smooth hypersurfaces of degree $\leq m$ in $\mathbb{P}^{m}$ are rationally connected.

DeFinition 15. - Let $Y$ be a smooth algebraic space of dimension $d$ and $f: \mathbb{P}^{1} \rightarrow Y$ a nonconstant morphism, so we have an isomorphism

$$
f^{*} T_{Y} \simeq \mathcal{O}_{\mathbb{P}^{1}}\left(a_{1}\right) \oplus \ldots \oplus \mathcal{O}_{\mathbb{P}^{1}}\left(a_{d}\right)
$$

for suitable integers $a_{1}, \ldots, a_{d}$. Then $f$ is free (resp. very free) if each $a_{i} \geq 0$ (resp. $\left.a_{i} \geq 1\right)$.

We recall some key properties:

(1) Let $Y$ be a proper rationally chain connected variety. Then any two closed points are contained in a connected curve with rational irreducible components, [11] IV.3.5.1. 
(2) If the ground field $k$ is uncountable then $Y$ is rationally connected (resp. rationally chain connected) if any two very general closed points $y_{1}$ and $y_{2}$ are contained in an irreducible rational curve (resp. connected curve with rational irreducible components), [11] IV.3.6.

(3) Let $Y$ be a smooth proper rationally connected variety, $y_{1}, \ldots, y_{m}$ points in $Y$, and $v_{i} \in T_{y_{i}} Y$ nonzero tangent vectors. Then there exists a very free morphism $f: \mathbb{P}^{1} \rightarrow Y$, an immersion (in the sense of differential geometry) if $\operatorname{dim}(Y)=2$ and an embedding if $\operatorname{dim}(Y) \geq 3$, so that the image contains $y_{1}, \ldots, y_{m}$ and is embedded at these points with the prescribed tangent vectors [11] IV.3.9 [8] 2.2 .

(4) A smooth variety $Y$ is rationally connected if it is rationally chain connected, [11] IV.3.10.3.

(5) Let $\pi: \mathcal{Y} \rightarrow B$ be a proper equidimensional morphism over an irreducible base. If the generic fiber of $\pi$ is rationally chain connected then every fiber is rationally chain connected, [11] IV.3.5.2.

(6) If $\pi: \mathcal{Y} \rightarrow B$ is a smooth proper morphism then the locus

$$
\left\{b \in B: \mathcal{Y}_{b} \text { is rationally connected }\right\}
$$

is open, [11] IV.3.11.

Properties (5) and (6) also hold for good models (which are only assumed to be algebraic spaces over $B)$.

EXAmPle 16. - Property (5) does not guarantee that every fiber is rationally connected: Consider the family of cubic surfaces

$$
\mathcal{X}:=\left\{(w, x, y, z ; t): x^{3}+y^{3}+z^{3}=t w^{3}\right\} \rightarrow \mathbb{A}_{t}^{1} .
$$

The generic fiber is rationally connected but the fiber $\mathcal{X}_{0}$ is a cone over an elliptic curve, which is not rationally connected.

3.2. Sketch of Theorem 3. - We outline the main issues in the proof of Theorem 3; details are given in Section 5. By Proposition 11, we are reduced to proving the existence of a section passing through specific smooth points $r_{i}$ of singular fibers of the iterated blow-up. Theorem 4 does not immediately imply this, but it does guarantee a section $\sigma$ passing through some point $x_{i}$ of each of these fibers. Property (5) of rationally connected varieties from Section 3.1 guarantees the existence of some chain $T_{i}$ of rational curves in the corresponding fiber joining $x_{i}$ and $r_{i}$. 
The difficulty is to choose these so that $C:=\sigma(B) \cup_{i \in I} T_{i}$ deforms to a section containing $r_{i}$, for each $i \in I$. In particular, it is necessary that $C$ intersect the fibral exceptional divisor $E_{i, N}$ containing $r_{i}$ in one point and not intersect the other components of the corresponding fiber; this constrains the homology class of $T_{i}$. Furthermore, we must describe each $T_{i}$ explicitly so the deformation space of $C$ can be analyzed.

\section{Deformation theory}

In this section, a curve is a connected reduced finite-type scheme of dimension one.

4.1. Combs. - Recall the dual graph associated with a nodal curve $C$ : Its vertices are indexed by the irreducible components of $C$ and its edges are indexed by the intersections of these components.

Definition 17. - A projective nodal curve $C$ is tree-like if

- each irreducible component of $C$ is smooth;

- the dual graph of $C$ is a tree.

We shall require a slight generalization of the standard notion of a comb (cf. [11]):

DeFinition 18. - A comb with $m$ reducible teeth is a projective nodal curve $C$ which is the union of $m+1$ subcurves $D, T_{1}, \ldots, T_{m}$ such that

$-D$ is smooth and irreducible;

$-T_{\ell} \cap T_{\ell^{\prime}}=\emptyset$, for all $\ell \neq \ell^{\prime}$;

- each $T_{\ell}$ meets $D$ transversally in a single point; and

- each $T_{\ell}$ is a chain of $\mathbb{P}^{1}$ 's.

Here $D$ is called the handle and the $T_{\ell}$ the teeth.

4.2. Vector bundle lemmas. - Let $C$ be a smooth curve. Here a 'vector bundle' $\mathcal{V}$ on $C$ designates a locally free sheaf and a 'subbundle' $\mathcal{U} \subset \mathcal{V}$ a subsheaf which is locally a direct summand; this is the case whenever $\mathcal{V} / \mathcal{U}$ is torsion-free.

Given a collection of distinct points $\mathfrak{q}=\left\{q_{1}, \ldots, q_{m}\right\} \subset C$ and onedimensional subspaces of the fibers $\xi_{q_{\ell}} \subset \mathcal{V}_{q_{\ell}}, \ell=1, \ldots, m$, there exists 
C

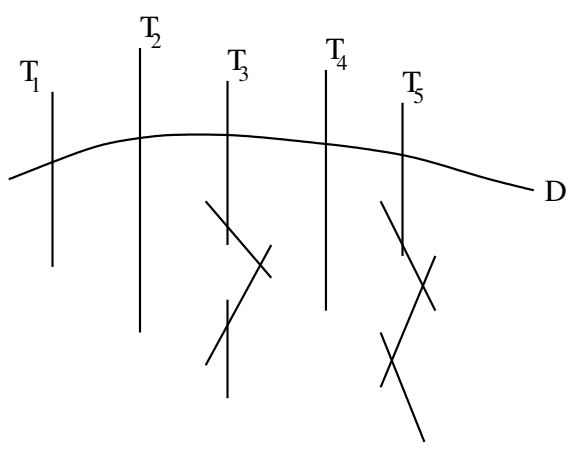

Figure 2. A comb with five reducible teeth

a rank-one subbundle $\mathcal{L} \subset \mathcal{V}$ with fiber at $q_{\ell}$ equal to $\xi_{q_{\ell}}, \ell=1, \ldots, m$. The extension

$$
0 \longrightarrow \mathcal{L} \longrightarrow \mathcal{L} \otimes \mathcal{O}_{C}(\mathfrak{q}) \longrightarrow \mathcal{L} \otimes\left(\mathcal{O}_{C}(\mathfrak{q})\right)_{\mathfrak{q}} \longrightarrow 0
$$

induces

$$
0 \longrightarrow \mathcal{V} \longrightarrow \mathcal{V}^{\prime} \longrightarrow Q \longrightarrow 0
$$

where $Q:=\left(\mathcal{L} \otimes \mathcal{O}_{C}(\mathfrak{q})\right)_{\mathfrak{q}}$ is supported on $\mathfrak{q}$ and has length one at each $q_{\ell}$. This extension depends on the $q_{\ell}$ and $\xi_{q_{\ell}}$ but not on $\mathcal{L}$. The saturation of $\mathcal{L}$ in $\mathcal{V}^{\prime}$ is isomorphic to $\mathcal{L} \otimes \mathcal{O}_{C}(\mathfrak{q})$.

Lemma 19. - Retain the notation introduced above. A subbundle $\mathcal{U} \subset$ $\mathcal{V}$ is also a subbundle of $\mathcal{V}^{\prime}$ if $\xi_{q_{\ell}} \cap \mathcal{U}_{q_{\ell}}=0$ for each $\ell$.

Proof. - We have exact sequences

$$
\begin{aligned}
& 0 \rightarrow \operatorname{Hom}\left(\mathcal{L} \otimes \mathcal{O}_{C}(\mathfrak{q}), \mathcal{V}\right) \rightarrow \operatorname{Hom}(\mathcal{L}, \mathcal{V}) \rightarrow \operatorname{Ext}^{1}(Q, \mathcal{V}) \\
& 0 \rightarrow \mathcal{H o m}\left(\mathcal{L} \otimes \mathcal{O}_{C}(\mathfrak{q}), \mathcal{V}\right) \rightarrow \mathcal{H o m}(\mathcal{L}, \mathcal{V}) \rightarrow \mathcal{E} x t^{1}(Q, \mathcal{V}) \rightarrow 0
\end{aligned}
$$

and the extension class $\eta_{\mathcal{V}^{\prime}} \in \operatorname{Ext}^{1}(Q, \mathcal{V})$ is the image of the inclusion $\mathcal{L} \hookrightarrow \mathcal{V}$ under the connecting homomorphism. Since $\mathcal{L}$ is saturated in $\mathcal{V}$ at $q_{\ell}, \eta_{V^{\prime}}$ localizes to a nonzero element of $\mathcal{E} x t^{1}(Q, \mathcal{V})_{q_{\ell}}$ for each $\ell$.

Since $\mathcal{U} \subset \mathcal{V}$ is a subbundle, $\mathcal{V} / \mathcal{U}$ has no torsion and thus is locally free. The class $\eta_{\mathcal{V}^{\prime}}$ naturally induces an extension

$$
0 \longrightarrow \mathcal{V} / \mathcal{U} \longrightarrow \mathcal{V}^{\prime} / \mathcal{U} \longrightarrow Q \rightarrow 0
$$


classified by $\eta_{\mathcal{V}^{\prime} / \mathcal{U}} \in \operatorname{Ext}^{1}(Q, \mathcal{V} / \mathcal{U})$, the image of the composition

$$
\mathcal{L} \hookrightarrow \mathcal{V} \rightarrow \mathcal{V} / \mathcal{U}
$$

under the connecting homomorphism. Our hypothesis guarantees that $\mathcal{L}$ is a subbundle of $\mathcal{V} / \mathcal{U}$ near $q_{\ell}$, hence $\eta_{\mathcal{V}^{\prime}} / \mathcal{U}$ localizes to a nonzero element of $\mathcal{E} x t^{1}(Q, \mathcal{V} / \mathcal{U})_{q_{\ell}}$ for each $\ell$. It follows that $\mathcal{V}^{\prime} / \mathcal{U}$ is torsion-free, so $\mathcal{U}$ is a subbundle.

Lemma 20 ([9], Lemma 2.5). — Retain the notation introduced above. Fix an integer $N$ and a vector bundle $\mathcal{V}$. Then there exist points $q_{1}, \ldots, q_{m}$ and one-dimensional subspaces $\xi_{q_{\ell}} \subset \mathcal{V}_{q_{\ell}}, \ell=1, \ldots, m$, such that

$$
H^{1}\left(\mathcal{V}^{\prime} \otimes \mathcal{O}_{C}\left(-w_{1}-\ldots-w_{N}\right)\right)=0
$$

for any points $w_{1}, \ldots, w_{N} \in C$.

Our next lemma is well known (cf. [14], Section 2) but we provide a proof for the convenience of the reader:

Lemma 21. - Let $C$ be a tree-like curve and $\mathcal{V}$ a vector bundle on $C$. If for each irreducible component $C_{\ell}$ of $C$ the restriction $\mathcal{V} \otimes \mathcal{O}_{C_{\ell}}$ is globally generated then $\mathcal{V}$ is globally generated. Furthermore,

$$
H^{1}(C, \mathcal{V}) \rightarrow \oplus_{\ell} H^{1}\left(\mathcal{V} \otimes \mathcal{O}_{C_{\ell}}\right)
$$

is an isomorphism.

Proof. - We do induction on the number of irreducible components; the case of one component is trivial. Otherwise, express $C$ as a union $D \cup D^{c}$, where $D$ is irreducible with connected complement in $C$ and $D^{c}=\overline{C \backslash D}$ is tree-like. Let $q$ be the node of $C$ joining $D$ and $D^{c}$,

$$
g: C^{\prime}:=D \amalg D^{c} \rightarrow C
$$

the partial normalization of $C$ at $q$, and $r, r^{c}$ the points of $C^{\prime}$ with $g(r)=$ $g\left(r^{c}\right)=q$. The descent data for $\mathcal{V}$ consist of the pullback $g^{*} \mathcal{V}$ and an isomorphism

$$
\phi:\left(g^{*} \mathcal{V}\right)_{r} \rightarrow\left(g^{*} \mathcal{V}\right)_{r^{c}}
$$

induced by identifications of fibers

$$
\left(g^{*} \mathcal{V}\right)_{r} \simeq \mathcal{V}_{q} \simeq\left(g^{*} \mathcal{V}\right)_{r^{c}}
$$


Recall the exact sequences relating the cohomology of $\mathcal{V}$ and $g^{*} \mathcal{V}$ :

$$
\begin{gathered}
0 \rightarrow H^{0}(C, \mathcal{V}) \rightarrow H^{0}\left(C^{\prime}, g^{*} \mathcal{V}\right) \rightarrow\left(g^{*} \mathcal{V}\right)_{r} \oplus\left(g^{*} \mathcal{V}\right)_{r c} \stackrel{(-\phi, \text { Id })}{\rightarrow}\left(g^{*} \mathcal{V}\right)_{r^{c}} \rightarrow 0 \\
H^{0}\left(C^{\prime}, g^{*} \mathcal{V}\right) \rightarrow\left(g^{*} \mathcal{V}\right)_{r} \oplus\left(g^{*} \mathcal{V}\right)_{r^{c}} \rightarrow H^{1}(C, \mathcal{V}) \rightarrow H^{1}\left(C^{\prime}, g^{*} \mathcal{V}\right) \longrightarrow 0
\end{gathered}
$$

By the inductive hypothesis, $g^{*} \mathcal{V}$ is globally generated on $C^{\prime}$. Since $r$ and $r^{c}$ are on different connected components of $C^{\prime}$, the second exact sequence guarantees that $H^{1}(C, \mathcal{V}) \rightarrow H^{1}\left(C^{\prime}, g^{*} \mathcal{V}\right)$ is injective; the cohomology statement follows. Since $g^{*} \mathcal{V}$ is globally generated, for each section over $D$ there exists a section over $D^{c}$ compatible under the isomorphism $\phi$, and vice versa. These compatible pairs of sections descend to elements of $H^{0}(C, \mathcal{V})$. Thus given $p \in D$ and $v \in \mathcal{V}_{p}$, a section $t \in H^{0}\left(D, \mathcal{V} \otimes \mathcal{O}_{D}\right)$ with $t(p)=v$ extends to a section over $C$.

4.3. Analysis of normal bundles. - We describe the normal bundle of a nodal curve immersed in a smooth algebraic space. Our main references are Section 2 of [9] and Section 6 of [1]. See [2] and [11] I.5 for foundational results on Hilbert 'schemes' of algebraic spaces, [11] II.1 for applications to morphisms of curves into spaces, and [10] for an extension of Theorem 1 to positive characteristic using this machinery.

If $C$ is a nodal curve embedded into a smooth space $Y$ then $\mathcal{N}_{C / Y}$ is defined as the dual to the kernel of the restriction homomorphism of Kähler differentials $\Omega_{Y}^{1} \otimes \mathcal{O}_{C} \rightarrow \Omega_{C}^{1}$; a local computation shows this is locally free. First order deformations of $C \subset Y$ are given by $H^{0}\left(C, \mathcal{N}_{C / Y}\right)$; obstructions are given by $H^{1}\left(C, \mathcal{N}_{C / Y}\right)$.

Let $D$ be a union of irreducible components of $C$ and $\mathfrak{q}=\left\{q_{1}, \ldots, q_{m}\right\}$ the locus where $D^{c}:=\overline{C \backslash D}$ meets $D$. At a node of $C$, the tangent cone is a union of two one-dimensional subspaces, the tangents to the transverse branches. The tangent to $D^{c}$ at $q_{\ell}$ yields a one-dimensional subspace

$$
\xi_{q_{\ell}} \subset\left(\mathcal{N}_{D / Y}\right)_{q_{\ell}}
$$

As in Section 4.2, these induce a natural extension $\mathcal{N}_{D / Y}^{\prime}$ of $\mathcal{N}_{D / Y}$, which coincides with the restriction to $D$ of the normal bundle to $C$ in $Y$

$$
0 \longrightarrow \mathcal{N}_{D / Y} \longrightarrow \mathcal{N}_{C / Y} \otimes \mathcal{O}_{D} \longrightarrow Q \longrightarrow 0 .
$$

Here $Q$ is a torsion sheaf supported on $\mathfrak{q}$, with length one at each point $q_{\ell}, \ell=1, \ldots, m$. Sections of $\mathcal{N}_{C / Y} \otimes \mathcal{O}_{D}$ can be interpreted as sections of $\mathcal{N}_{D / Y}$ with poles at the $q_{\ell}$ in the directions $T_{q_{\ell}} D^{c}$. 
We shall need a slight generalization: We continue to assume that $C$ is a nodal curve and $Y$ is nonsingular. Let $f: C \rightarrow Y$ denote an immersion whose image is a nodal curve. The restriction homomorphism $f^{*} \Omega_{Y}^{1} \rightarrow$ $\Omega_{C}^{1}$ is surjective and the dual to its kernel is still locally free. This is denoted $\mathcal{N}_{f}$ and coincides with $\mathcal{N}_{C / Y}$ when $f$ is an embedding. First order deformations of $f: C \rightarrow Y$ are given by $H^{0}\left(C, \mathcal{N}_{f}\right)$; obstructions are given by $H^{1}\left(C, \mathcal{N}_{f}\right)$. This set-up differs from the standard deformation theory of morphisms in that we ignore reparametrizations of $C$. When $D$ is a union of irreducible components of $C$ as above, then the analogous extension takes the form (cf. Lemma 2.6 of [9]):

$$
0 \longrightarrow \mathcal{N}_{\left.f\right|_{D}} \longrightarrow \mathcal{N}_{f} \otimes \mathcal{O}_{D} \longrightarrow Q \longrightarrow 0
$$

This analysis gives the following infinitesimal smoothing criterion: (cf. Lemma 2.6 of [9]):

LEMMA 22. - Retain the above notation. A first order deformation $t \in H^{0}\left(C, \mathcal{N}_{f}\right)$ smooths the node $q_{\ell}$ to first order if the restriction $\left.t\right|_{D} \in$ $\mathcal{N}_{f} \otimes \mathcal{O}_{D}$ is nonzero in $Q_{q_{\ell}}$. In particular, each deformation of $f$ over the spectrum of a DVR with tangent vector $t$ smooths $q_{\ell}$.

Proposition 23. - Let $Y$ be a smooth algebraic space, $E \subset Y$ a smooth subspace of codimension one, $C$ a nodal curve, $D \subset C$ a union of irreducible components of $C, D^{c}=\overline{D \backslash C}$, and $\mathfrak{q}=\left\{q_{\ell}\right\}=D \cap D^{c}$. Let $f: C \rightarrow Y$ be an immersion with image a nodal curve so that $f(D) \subset E$, $f(D \backslash C) \subset Y \backslash E$, and $f\left(D^{c}\right)$ is transverse to $E$ at each point of $f(\mathfrak{q})$. If $g: D \rightarrow E$ is the resulting immersion of $D$ into $E$ then $\mathcal{N}_{g}$ is saturated in $\mathcal{N}_{f} \otimes \mathcal{O}_{D}$ and we have the following diagram: 


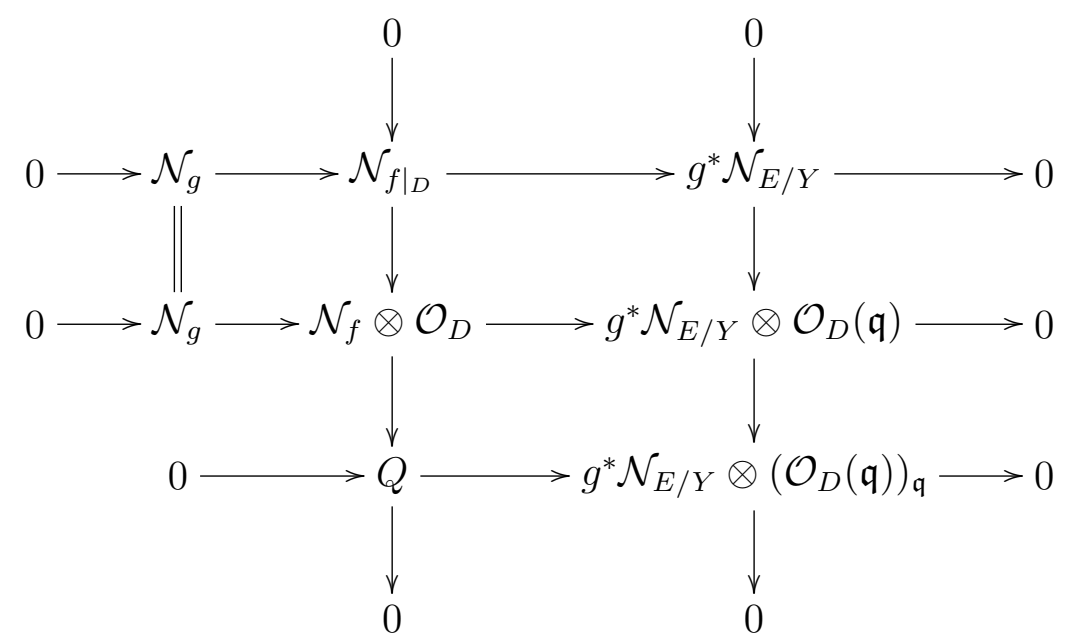

Proof. - For each $\ell$ the composition

$$
T_{q_{\ell}} D^{c} \rightarrow T_{q_{\ell}} Y \rightarrow T_{q_{\ell}} Y / T_{q_{\ell}} D
$$

determines a one-dimensional subspace $\xi_{\ell} \subset\left(\mathcal{N}_{\left.f\right|_{D}}\right)_{q_{\ell}}$. The transversality hypothesis implies $\left(\mathcal{N}_{g}\right)_{q_{\ell}} \cap \xi_{q_{\ell}}=0$ in $\left(\mathcal{N}_{\left.f\right|_{D}}\right)_{q_{\ell}}$. Using Lemma 19, we conclude that $\mathcal{N}_{\left.f\right|_{D}}$ is a subbundle in $\mathcal{N}_{f} \otimes \mathcal{O}_{D}$, so the quotient

$$
R=\left(\mathcal{N}_{f} \otimes \mathcal{O}_{D}\right) / \mathcal{N}_{\left.f\right|_{D}}
$$

is locally free. This sheaf arises as an extension

$$
0 \longrightarrow g^{*} \mathcal{N}_{E / Y} \longrightarrow R \longrightarrow Q \longrightarrow 0,
$$

where $Q$ is supported on $\mathfrak{q}$ with length one at each $q_{\ell}$. We may therefore identify extension (4.1) with the tensor product of $g^{*} \mathcal{N}_{E / Y}$ with

$$
0 \longrightarrow \mathcal{O}_{D} \longrightarrow \mathcal{O}_{D}(\mathfrak{q}) \longrightarrow\left(\mathcal{O}_{D}(\mathfrak{q})\right)_{\mathfrak{q}} \longrightarrow 0
$$

Proposition 24. - Let $C$ be a tree-like curve, $Y$ a smooth algebraic space, and $f: C \rightarrow Y$ an immersion with nodal image. Suppose that for each irreducible component $C_{\ell}$ of $C, H^{1}\left(C_{\ell}, \mathcal{N}_{f} \otimes \mathcal{O}_{C_{\ell}}\right)=0$ and $\mathcal{N}_{f} \otimes \mathcal{O}_{C_{\ell}}$ is globally generated. Then $f: C \rightarrow Y$ deforms to an immersion of a smooth curve into $Y$.

Suppose furthermore that $\mathfrak{p}=\left\{p_{1}, \ldots, p_{w}\right\} \subset C$ is a collection of smooth points such that for each component $C_{\ell} H^{1}\left(\mathcal{N}_{f} \otimes \mathcal{O}_{C_{\ell}}(-\mathfrak{p})\right)=0$ 
and the sheaf $\mathcal{N}_{f} \otimes \mathcal{O}_{C_{\ell}}(-\mathfrak{p})$ is globally generated. Then $f: C \rightarrow Y$ deforms to an immersion of a smooth curve into $Y$ containing $f(\mathfrak{p})$.

Proof. - Our argument is similar to the constructions of Section 2 of [9] and Lemma 65 of [1]. Lemma 21 implies that $H^{1}\left(C, \mathcal{N}_{f}\right)=0$ and $\mathcal{N}_{f}$ is globally generated. Hence the space of maps is unobstructed and every first-order deformation of $f$ lifts to a deformation over the spectrum of a DVR. Global generation implies the existence of $t \in H^{0}\left(C, \mathcal{N}_{f}\right)$ so that, for each component $C_{\ell}$, the image of $t$ in

$$
H^{0}\left(C_{\ell}, Q\left(C_{\ell}\right)\right), \quad Q\left(C_{\ell}\right):=\left(\mathcal{N}_{f} \otimes \mathcal{O}_{C_{\ell}}\right) / \mathcal{N}_{\left.f\right|_{C_{\ell}}}
$$

is nonzero at each point of the support of $Q\left(C_{\ell}\right)$. The first-order deformation $t$ smooths each node of $C$ by Lemma 22 .

For the second part, consider those maps with image containing $f(\mathfrak{p})$. Our cohomology assumption guarantees that this space is unobstructed; in addition, $\mathcal{N}_{f} \otimes \mathcal{O}_{C}(-\mathfrak{p})$ is globally generated. Hence there exists a $u \in H^{0}\left(\mathcal{N}_{f} \otimes \mathcal{O}_{C}(-\mathfrak{p})\right)$ so that, for every component $C_{\ell}$, the image of $u$ in $Q\left(C_{\ell}\right)$ is nonzero at each point of its support. Note that

$$
\left(\mathcal{N}_{f} \otimes \mathcal{O}_{C_{\ell}}(-\mathfrak{p})\right) /\left(\mathcal{N}_{\left.f\right|_{C_{\ell}}} \otimes \mathcal{O}_{C_{\ell}}(-\mathfrak{p})\right)=\left(\mathcal{N}_{f} \otimes \mathcal{O}_{C_{\ell}}\right) / \mathcal{N}_{\left.f\right|_{C_{\ell}}}
$$

since the quotient is a torsion sheaf with support disjoint from $\mathfrak{p}$. Hence the first-order deformation $u$ smooths each node of $C$ and contains $f(\mathfrak{p})$.

\section{Proof of the main theorem}

The theorem is well known when $d=\operatorname{dim}(X)=1$. The only smooth proper rationally connected curve is $\mathbb{P}^{1}$, which satisfies weak approximation. We may therefore assume $d \geq 2$.

Recall the set-up of Proposition 11: It suffices to show that for each integer $N$, finite set $\left\{b_{i}\right\}_{i \in I} \subset B \backslash S$, and collection of $N$-jet data $J$ supported in the fibers over $\left\{b_{i}\right\}_{i \in I}$, there exists a section in the iterated blowup $\mathcal{X}(J)$ passing through prescribed points $r_{i} \in E_{i, N} \backslash E_{i, N-1}$.

We proceed by induction on $N$; the base case $N=0$ is essentially Theorem 4. However, our assumptions are slightly weaker: We are not assuming $\mathcal{X}$ is a scheme. 
The total space of $\mathcal{X}$ is smooth along the fibers $\mathcal{X}_{b_{i}}, i \in I$, so we may resolve the singularities of $\mathcal{X}$ without altering these fibers. Theorem 1 gives a section $\sigma$ of $\pi$. Let $q_{i}=\sigma\left(b_{i}\right)$ for each $i \in I$; let $I^{\prime} \subset I$ (resp. $\left.I^{\prime \prime} \subset I\right)$ denote those indices with $q_{i} \neq r_{i}\left(\operatorname{resp} . q_{i}=r_{i}\right)$.

Lemma 25. - There exists a comb $C$ with handle $\sigma(B)$ and smooth teeth $T_{1}, \ldots, T_{m}$ and an immersion $f: C \rightarrow \mathcal{X}$ with nodal image so that:

- the $T_{\ell}$ are free rational curves in distinct smooth fibers of $\pi$;

- for each $i \in I^{\prime}$, there is a tooth $T_{i}$ containing $r_{i}$ as a smooth point;

- let $\mathfrak{r}$ denote the sum of the points of $C$ mapping to the $r_{i}, i \in I$; then the restriction of $\mathcal{N}_{f} \otimes \mathcal{O}_{C}(-\mathfrak{r})$ to each irreducible component of $C$ is globally generated and has no higher cohomology.

We emphasize that $f$ can be taken to be an embedding if $d>2$. Proposition 24 implies $f: C \rightarrow \mathcal{X}$ admits a deformation $\tilde{f}: \tilde{C} \rightarrow \mathcal{X}$, where $\tilde{C}$ is smooth and $\tilde{f}(\tilde{C})$ contains each of the $r_{i}$. Since all the teeth $T_{1}, \ldots, T_{m}$ are contained in fibers of $\mathcal{X} \rightarrow B, C$ intersects the generic fiber in one point. Thus the deformed curve $\tilde{C}$ also meets the generic fiber in one point and hence is a section of $\mathcal{X} \rightarrow B$.

Proof. - Here are the details of the construction of the comb of Lemma 25. For each $\ell$

$$
f_{\ell}: T_{\ell} \rightarrow \mathcal{X}_{b_{\ell}}
$$

is a free rational curve with nodal image, so that $\sigma\left(b_{\ell}\right) \in f_{\ell}\left(T_{\ell}\right)$ as a smooth point. For $\ell=1, \ldots,\left|I^{\prime}\right|$ we choose these so that $r_{i}$ is contained in the image as a smooth point. For $\ell=\left|I^{\prime}\right|+1, \ldots, m$, we choose these in generic fibers of good reduction with generic tangent directions $\xi_{\ell} \subset$ $T_{\sigma\left(b_{\ell}\right)} \mathcal{X}_{b_{\ell}}$ satisfying the hypotheses of Lemma 20, so that the extension

$$
0 \longrightarrow \mathcal{N}_{\sigma(B)} \longrightarrow \mathcal{N}_{f} \otimes \mathcal{O}_{\sigma(B)} \longrightarrow Q(\sigma(B)) \longrightarrow 0
$$

is globally-generated and has no higher cohomology, even after twisting by $\mathcal{O}_{\sigma(B)}\left(-\sum_{i \in I^{\prime \prime}} r_{i}\right)$.

We next address the inductive step. Let $J^{\prime}$ denote order- $(N-1)$ truncation of $J$, i.e., $j_{i}^{\prime}$ is the restriction of $j_{i}$ to $\operatorname{Spec}\left(\mathcal{O}_{B, b_{i}} / \mathfrak{m}_{B, b_{i}}^{N}\right)$ for each $i$. The inductive hypothesis applied to $J^{\prime}$ guarantees the existence of a section $s^{\prime}: B \rightarrow \mathcal{X}\left(J^{\prime}\right)$ passing through arbitrary points

$$
r_{i}^{\prime} \in\left(E_{i, N-1}^{\prime} \backslash E_{i, N-2}^{\prime}\right) \subset \mathcal{X}\left(J^{\prime}\right) .
$$


Specifically, we choose $s^{\prime}$ so that it has jet data $J^{\prime}$ over the points $\left\{b_{i}\right\}_{i \in I}$. Let $\sigma: B \rightarrow \mathcal{X}(J)$ denote the proper transform of $s^{\prime}$ in $\mathcal{X}(J)$. By construction, $\sigma$ meets $\mathcal{X}(J)_{b_{i}}$ in a point $q_{i} \in E_{i, N} \backslash E_{i, N-1}$ for each $i$. Our goal is to find a section $s: B \rightarrow \mathcal{X}(J)$ such that for each $i \in I$ and $r_{i} \in E_{i, N} \backslash E_{i, N-1}, s\left(b_{i}\right)=r_{i}$. Again, $I^{\prime} \subset I$ (resp. $\left.I^{\prime \prime} \subset I\right)$ denotes those indices with $q_{i} \neq r_{i}\left(\operatorname{resp} . q_{i}=r_{i}\right)$.

Lemma 26. - There exists a comb $C$ with handle $\sigma(B)$ and reducible teeth $T_{1}, \ldots, T_{m}$ and an immersion $f: C \rightarrow \mathcal{X}(J)$ with nodal image so that:

- for each $i \in I^{\prime}$, there is a tooth $T_{i}$ mapped to $\mathcal{X}(J)_{b_{i}}$ and containing $r_{i}$;

- $C$ is smoothly embedded at $r_{i}$ for each $i \in I^{\prime}$, so there is a unique component $T_{i, N} \subset C$ containing $r_{i}$;

- the remaining teeth $T_{\left|I^{\prime}\right|+1}, \ldots, T_{m}$ are free rational curves contained in generic fibers of $\mathcal{X}(J) \rightarrow B$ of good reduction;

- the restriction of $\mathcal{N}_{f} \otimes \mathcal{O}_{C}(-\mathfrak{r})$ to each irreducible component is globally generated and has no higher cohomology.

Again, $f$ can be taken to be an embedding if $d>2$. Proposition 24 implies that $f: C \rightarrow \mathcal{X}(J)$ admits a deformation $\tilde{f}: \tilde{C} \rightarrow \mathcal{X}(J)$, where $\tilde{C}$ is smooth and $\tilde{f}(\tilde{C})$ contains each of the $r_{i}$. The image $\tilde{f}(\tilde{C})$ is the desired section of $\mathcal{X}(J) \rightarrow B$. Hence Theorem 3 is reduced to proving this lemma.

Proof. - We start the proof of Lemma 26 by describing the teeth $T_{i}$ with $i \in I^{\prime}$. Recall from Section 2.3 that $E_{i, N} \simeq \mathbb{P}^{d}$ and $E_{i, N} \cap E_{i, N-1}$ is a hyperplane section in this $\mathbb{P}^{d}$. Let $T_{i, N}$ denote the unique line joining $r_{i}$ to $q_{i}=\sigma\left(b_{i}\right)$; let $q_{i, N-1}$ denote the intersection of this line with $E_{i, N-1}$. We have

$$
E_{i, N-1} \simeq \mathrm{Bl}_{r_{i, N-1}} \mathbb{P}^{d}
$$

with exceptional divisor $E_{i, N-1} \cap E_{i, N} \simeq \mathbb{P}^{d-1}$; there is a unique line in $\mathbb{P}^{d}$ containing $r_{i, N-1}$ whose proper transform $T_{i, N-1} \subset E_{i, N-1}$ meets $q_{i, N-1}$. Let $q_{i, N-2}$ denote the intersection of this line with $E_{i, N-2}$. Continuing in this way, we obtain a sequence of embedded smooth rational curves

$$
T_{i, n} \subset E_{i, n} \simeq \mathrm{Bl}_{r_{i, n}} \mathbb{P}^{d}, \quad n>0,
$$


each the proper transform of a line meeting $r_{i, n}$. Let $q_{i, 0}$ denote the intersection of $T_{i, 1}$ with $E_{i, 0}$, which is a point in the exceptional divisor of

$$
E_{i, 0}=\mathrm{Bl}_{r_{i, 0}} \rightarrow \mathcal{X}_{b_{i}} .
$$

Let $g_{i, 0}: T_{i, 0} \rightarrow E_{i, 0}$ be a free rational curve, immersed so that the image is a nodal curve, with $q_{i, 0} \in g_{i, 0}\left(T_{i, 0}\right)$ as a smooth point. Property (3) of rationally connected varieties gives such a curve; $g_{i, 0}$ can be taken to be an embedding when $d>2$. Let $f_{i, 0}$ denote the composition of $g_{i, 0}$ with the inclusion $E_{i, 0} \subset \mathcal{X}(J)$, and

$$
f_{i}: T_{i}=T_{i, 0} \cup \ldots \cup T_{i, N} \longrightarrow \mathcal{X}(J)
$$

the resulting map of the tooth into $\mathcal{X}(J)$.

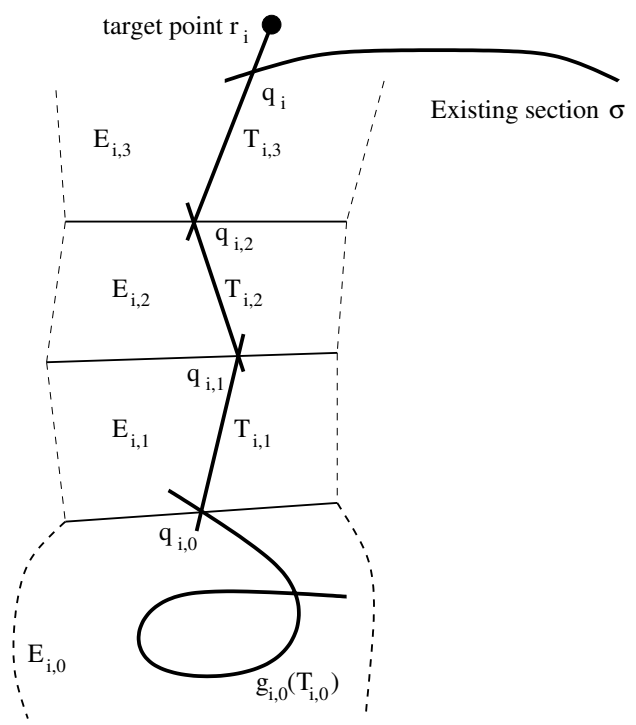

FiguRE 3. Attaching reducible teeth and moving the section

Sublemma 27. - The condition on normal bundles stipulated in Lemma 26 is satisfied along the reducible teeth $T_{i}, i \in I^{\prime}$. Precisely, we have

$$
\mathcal{N}_{f} \otimes \mathcal{O}_{T_{i, n}}= \begin{cases}\mathcal{O}_{\mathbb{P}^{1}}(+1)^{d} & \text { for } n=N \\ \mathcal{O}_{\mathbb{P}^{1}}^{d} & \text { for } n=1, \ldots, N-1 . \\ \oplus_{w=1}^{d-1} \mathcal{O}_{\mathbb{P}^{1}}\left(a_{w}\right) \oplus \mathcal{O}_{\mathbb{P}^{1}}, \quad a_{w} \geq 0 & \text { for } n=0\end{cases}
$$


Proof. — First, observe that

$$
\begin{aligned}
& \mathcal{N}_{T_{i, n} / E_{i, n}} \simeq \begin{cases}\mathcal{O}_{\mathbb{P}^{1}}(+1)^{d-1} & \text { for } n=N \\
\mathcal{O}_{\mathbb{P}^{1}}^{d-1} & \text { for } n=1, \ldots, N-1\end{cases} \\
& \mathcal{N}_{g_{i, 0}} \simeq \oplus_{w=1}^{d-1} \mathcal{O}_{\mathbb{P}^{1}}\left(a_{w}\right), \quad a_{w} \geq 0 ;
\end{aligned}
$$

the $a_{w}$ are nonnegative because $\mathcal{N}_{g_{i, 0}}$ is a quotient of $g_{i, 0}^{*} T_{E_{i, 0}}$, which is nonnegative. Fibers of $\mathcal{X}(J) \rightarrow B$ restrict to the zero divisor on each $T_{i, n}$ and $\sum_{n=0}^{N} E_{i, n}$ is equivalent to the class of a fiber, hence

$$
E_{i, n}\left|T_{i, n}=\left(-\sum_{n^{\prime} \neq n} E_{i, n^{\prime}}\right)\right| T_{i, n}
$$

It follows that

$\mathcal{N}_{E_{i, n} / \mathcal{X}(J)} \otimes \mathcal{O}_{T_{i, n}}=\mathcal{O}_{\mathcal{X}(J)}\left(E_{i, n}\right) \otimes \mathcal{O}_{T_{i, n}} \simeq \begin{cases}\mathcal{O}_{\mathbb{P}^{1}}(-1) & \text { for } n=N \\ \mathcal{O}_{\mathbb{P}^{1}}(-2) & \text { for } n=1, \ldots, N-1 \\ \mathcal{O}_{\mathbb{P}^{1}}(-1) & \text { for } n=0 .\end{cases}$

For $n>0$ we have the exact sequence

$$
0 \longrightarrow \mathcal{N}_{T_{i, n} / E_{i, n}} \longrightarrow \mathcal{N}_{T_{i, n} / \mathcal{X}(J)} \longrightarrow \mathcal{N}_{E_{i, n} / \mathcal{X}(J)} \otimes \mathcal{O}_{T_{i, n}} \longrightarrow 0,
$$

which splits in our situation. Therefore, we find

$$
\mathcal{N}_{T_{i, n} / \mathcal{X}(J)} \simeq\left\{\begin{array}{ll}
\mathcal{O}_{\mathbb{P}^{1}}(+1)^{d-1} \oplus \mathcal{O}_{\mathbb{P}^{1}}(-1) & \text { for } n=N \\
\mathcal{O}_{\mathbb{P}^{1}}^{d-1} \oplus \mathcal{O}_{\mathbb{P}^{1}}(-2) & \text { for } n=1, \ldots, N-1
\end{array} .\right.
$$

For $n=0$ we have

$$
0 \longrightarrow \mathcal{N}_{g_{i, 0}} \longrightarrow \mathcal{N}_{f_{i, 0}} \longrightarrow g_{i, 0}^{*} \mathcal{N}_{E_{i, 0} / \mathcal{X}(J)} \longrightarrow 0,
$$

which implies

$$
\mathcal{N}_{f_{i, 0}} \simeq \oplus_{w=1}^{d-1} \mathcal{O}_{\mathbb{P}^{1}}\left(a_{w}\right) \oplus \mathcal{O}_{\mathbb{P}^{1}}(-1)
$$

On first examination, the negative summands would make it hard to satisfy the requirements of Lemma 26. However, the nodes in the reducible teeth give enough positivity to overcome the negative factors. We use Proposition 23 to analyze the relationship between the normal bundles to the $T_{i, n}$ and the restriction to the normal bundle of the comb to these components.

When $n>0$, we have an exact sequence

$$
0 \longrightarrow \mathcal{N}_{T_{i, n} / \mathcal{X}(J)} \longrightarrow \mathcal{N}_{f} \otimes \mathcal{O}_{T_{i, n}} \longrightarrow Q\left(T_{i, n}\right) \longrightarrow 0,
$$


where $Q\left(T_{i, n}\right)$ is a torsion sheaf, supported at the nodes of $C$ on $T_{i, n}$. However, the positive summands of $\mathcal{N}_{T_{i, n} / \mathcal{X}(J)}$ are saturated in $\mathcal{N}_{f}$; only the negative summand fails to be saturated. When $n=1, \ldots, N-1$, the negative summand is isomorphic to $\mathcal{O}_{\mathbb{P}^{1}}(-2)$ and $Q\left(T_{i, n}\right)$ has length two, so the saturation is $\mathcal{O}_{\mathbb{P}^{1}}$. When $n=N$, the negative summand is isomorphic to $\mathcal{O}_{\mathbb{P}^{1}}(-1)$ and $Q\left(T_{i, N}\right)$ has length two and support $\left\{r_{i}, q_{i, N-1}\right\}$, so the saturation is $\mathcal{O}_{\mathbb{P}^{1}}$. When $n=0$ we have

$$
0 \longrightarrow \mathcal{N}_{f_{i, 0}} \longrightarrow \mathcal{N}_{f_{i}} \otimes \mathcal{O}_{T_{i, 0}} \longrightarrow Q\left(T_{i, 0}\right) \longrightarrow 0,
$$

with $Q\left(T_{i, 0}\right)$ of length one and supported at $q_{i, 0}$. The negative summand of $\mathcal{N}_{f_{i, 0}}$ is isomorphic to $\mathcal{O}_{\mathbb{P}^{1}}(-1)$, so the extension above induces

$$
0 \rightarrow \mathcal{O}_{\mathbb{P}^{1}}(-1) \rightarrow \mathcal{O}_{\mathbb{P}^{1}} \rightarrow Q\left(T_{i, 0}\right) \rightarrow 0,
$$

i.e., the saturation of the negative factor is $\mathcal{O}_{\mathbb{P}^{1}}$.

For $\ell=\left|I^{\prime}\right|+1, \ldots, m$ we take

$$
f_{\ell}: T_{\ell} \rightarrow \mathcal{X}_{b_{\ell}}
$$

to be free rational curves, immersed in generic fibers of good reduction so that the images are nodal, with $\sigma\left(b_{\ell}\right) \in f_{\ell}\left(T_{\ell}\right)$ as a smooth point. We choose these with generic tangent directions $\xi_{\ell} \subset T_{\sigma\left(b_{\ell}\right)} \mathcal{X}_{b_{\ell}}$ so that Lemma 20 guarantees $\mathcal{N}_{f} \otimes \mathcal{O}_{\sigma(B)}\left(-\sum_{i \in I^{\prime \prime}} r_{i}\right)$ is globally-generated and has no higher cohomology.

REMARK 28 (Some remarks on positive characteristic)

Much of our argument applies without change even when the ground field $k$ has positive characteristic. Here are the places where we used characteristic zero:

1. At the beginning of our argument, we require an initial section of $\pi$. The main Theorem of [10] guarantees such a section, provided the geometric fiber is smooth proper and separably rationally connected.

2. To apply our deformation theory, we require that the initial section be in the smooth locus of $\mathcal{X}$; we resolved singularities of $\mathcal{X}$ along the section to achieve this. Alternately, we could restrict to the open subset $B \backslash S \subset B$ of places of good reduction, working on a model over $B \backslash S$. However, we would then have to rework our deformation theory, doing everything relative to this open subset. 
3. Property (3) of Section 3.1 does not hold for arbitrary rationally connected varieties in positive characteristic; however, it is valid for separably rationally connected varieties.

Recall that a smooth variety $X$ is separably rationally connected if it admits a very free rational curve [1], item 35.

We say that a place of $F$ is of very good reduction for $X$ if $X$ admits a local model with smooth, separably rationally connected reduction at that place. By standard deformation theory, the existence of a place of very good reduction guarantees that $X$ is geometrically separably rationally connected over $F$.

This discussion suggests the following characteristic-free generalization of Theorem 3: A smooth proper geometrically separably rationally connected variety satisfies weak approximation at places of very good reduction.

\section{References}

[1] C. Araujo and J. Kollár - "Rational curves on varieties", Higher dimensional varieties and rational points (Budapest, 2001) (K. J. Böröczky, J. Kollár and S. T., eds.), Bolyai Soc. Math. Stud., vol. 12, Springer, Berlin, 2003, p. 13-68.

[2] M. Artin - "Algebraization of formal moduli. I", Global Analysis (Papers in Honor of K. Kodaira), Univ. Tokyo Press, Tokyo, 1969, p. 21-71.

[3] _ "Algebraization of formal moduli. II. Existence of modifications", Ann. of Math. (2) 91 (1970), p. 88-135.

[4] E. BrieskorN - "Die Auflösung der rationalen Singularitäten holomorpher Abbildungen", Math. Ann. 178 (1968), p. 255-270.

[5] _ "Singular elements of semi-simple algebraic groups", Actes du Congrès International des Mathématiciens (Nice, 1970), Tome 2, Gauthier-Villars, Paris, 1971, p. 279-284.

[6] F. Campana - "Connexité rationnelle des variétés de Fano", Ann. Sci. École Norm. Sup. (4) 25 (1992), no. 5, p. 539-545.

[7] J. L. Colliot-Thélène and P. Gille - "Remarques sur l'approximation faible sur un corps de fonctions d'une variable", Arithmetic of higher-dimensional algebraic varieties (Palo Alto, CA, 
2002), Progr. Math., vol. 226, Birkhäuser Boston, Boston, MA, 2004, p. 121-134.

[8] O. Debarre - "Variétés rationnellement connexes (d'après T. Graber, J. Harris, J. Starr et A. J. de Jong)", Astérisque 290 (2003), p. Exp. No. 905, ix, 243-266, Séminaire Bourbaki. Vol. 2001/2002.

[9] T. Graber, J. Harris and J. Starr - "Families of rationally connected varieties", J. Amer. Math. Soc. 16 (2003), no. 1, p. 57-67 (electronic).

[10] A. J. DE Jong and J. STARR - "Every rationally connected variety over the function field of a curve has a rational point", Amer. J. Math. 125 (2003), no. 3, p. 567-580.

[11] J. Kollár - Rational curves on algebraic varieties, Ergebnisse der Math., vol. 32, Springer-Verlag, Berlin, 1996.

[12] J. Kollár, Y. MiyaOKA and S. Mori - "Rationally connected varieties", J. Algebraic Geom. 1 (1992), no. 3, p. 429-448.

[13] D. MAdorE - "Approximation faible aux places de bonne réduction sur les surfaces cubiques sur les corps des fonctions", 2004, preprint.

[14] D. S. Nagaraj and C. S. Seshadri - "Degenerations of the moduli spaces of vector bundles on curves. II. Generalized Gieseker moduli spaces", Proc. Indian Acad. Sci. Math. Sci. 109 (1999), no. 2, p. $165-201$.

[15] M. NAGATA - "A generalization of the imbedding problem of an abstract variety in a complete variety", J. Math. Kyoto Univ. 3 (1963), p. 89-102. 